\title{
Comparison of Clinical Features and Outcomes of Medically Attended COVID-19 and Influenza Patients in a Defined Population in the 2020 Respiratory Virus Season
}

OPEN ACCESS

Edited by:

Fabrizio Ricci,

University of Studies G. d'Annunzio

Chieti and Pescara, Italy

Reviewed by:

Quanxin Long,

Chongqing Medical University, China Sunny Oteikwu Ochigbo, University of Calabar, Nigeria

Yiwu Yan,

Cedars-Sinai Medical Center,

United States

*Correspondence:

Jing Yang

yangjing780228@foxmail.com

Zhixin Liu

Izx20022456@126.com

†These authors have contributed equally to this work

Specialty section

This article was submitted to Infectious Diseases - Surveillance,

Prevention and Treatment a section of the journal

Frontiers in Public Health

Received: 01 October 2020 Accepted: 22 February 2021

Published: 23 March 2021

Citation:

Liu L, Zeng F, Rao J, Yuan S, Ji M, Lei X, Xiao X, Li Z, Li X, Du W, LiU Y Tan H, Li J, Zhu J, Yang J and Liu Z

(2021) Comparison of Clinical Features and Outcomes of Medically Attended COVID-19 and Influenza Patients in a Defined Population in the 2020 Respiratory Virus Season.

Front. Public Health 9:587425. doi: 10.3389/fpubh.2021.587425
Long Liu ${ }^{1,2,3+}$, Feng Zeng ${ }^{1,2+}$, Jingjing Rao ${ }^{1,2}$, Shengren Yuan ${ }^{1,2}$, Manshan Ji ${ }^{1,2}$, Xu Lei ${ }^{1,2}$, Xiao Xiao ${ }^{1,2}$, Zhijun $\mathrm{Li}^{1,2}$, Xiaohua $\mathrm{Li}^{4}$, Weixing $\mathrm{Du}^{1,2}$, Yanqing Liu ${ }^{1,2}$, Huabing Tan $^{1,2}$, Junmin $\mathrm{Li}^{1,2}$, Jianyong Zhu ${ }^{1,2}$, Jing Yang ${ }^{1,2 *}$ and Zhixin Liu ${ }^{1,2,3 *}$

1 Department of Infectious Diseases, Renmin Hospital, School of Basic Medical Sciences, Hubei University of Medicine, Shiyan, China, ${ }^{2}$ Department of Respiratory, Renmin Hospital, School of Basic Medical Sciences, Hubei University of Medicine, Shiyan, China, ${ }^{3}$ Hubei Key Laboratory of Embryonic Stem Cell Research, Hubei University of Medicine, Shiyan, China, ${ }^{4}$ Department of Emergency, Dongfeng Maojian Hospital, Sinopharm Group Corporation, Shiyan, China

The emergence of severe acute respiratory syndrome coronavirus 2 (SARS-COV-2), which is causing the coronavirus disease-2019 (COVID-19) pandemic, poses a global health threat. However, it is easy to confuse COVID-19 with seasonal influenza in preliminary clinical diagnosis. In this study, the differences between influenza and COVID-19 in epidemiological features, clinical manifestations, comorbidities and pathogen biology were comprehensively compared and analyzed. SARS-CoV-2 causes a higher proportion of pneumonia (90.67 vs. 17.07\%) and acute respiratory distress syndrome (12.00 vs. 0\%) than influenza A virus. The proportion of leukopenia for influenza patients was $31.71 \%$ compared with $12.00 \%$ for COVID-19 patients $(P=0.0096)$. The creatinine and creatine kinase were significantly elevated when there were COVID-19 patients. The basic reproductive number $\left(R_{0}\right)$ for SARS-CoV-2 is 2.38 compared with 1.28 for seasonal influenza A virus. The mutation rate of SARS-CoV-2 ranges from 1.12 $\times 10^{-3}$ to $6.25 \times 10^{-3}$, while seasonal influenza virus has a lower evolutionary rate $\left(0.60-2.00 \times 10^{-6}\right)$. Overall, this study compared the clinical features and outcomes of medically attended COVID-19 and influenza patients. In addition, the S477N and N439K mutations on spike may affect the affinity with receptor ACE2. This study will contribute to COVID-19 control and epidemic surveillance in the future.

Keywords: influenza, COVID-19, epidemic, comparison, adaptive mutation

\section{INTRODUCTION}

An increasing number of COVID-19 cases have caused a global health burden due to the rapid transmission throughout the human community. The pathogen SARS-COV-2 causes respiratory system and severe systemic symptoms through respiratory tract infection $(1,2)$. As of February 17th, 2021, more than 100 million COVID-19 cases were confirmed, and more than 2 million deaths had occurred (3). Although many countries are developing vaccines and racing to run clinical trials 
(4), there are still many unresolved questions regarding viral invasion, pathogenesis and clinical features. In particular, the relationship between mutations and pathogenicity or transmissibility remains unknown.

The seasonal influenza epidemic is caused by strains of influenza virus, including two influenza $A$ viruses $(\mathrm{H} 1 \mathrm{~N} 1$ and $\mathrm{H} 3 \mathrm{~N} 2$ ) and one influenza $\mathrm{B}$ virus. There have been four documented influenza pandemics in the past 100 years (in 1918, 1957, 1968, and 2009) (5). The influenza case fatality rates of the 1918 and $2009 \mathrm{H} 1 \mathrm{~N} 1$ pandemics ranged from $0.1 \%$ to $2.5 \%$ $(6,7)$. The very important issue is the emergence of a new subtype or strain through uncontrollable and unpredictable mutations or antigenic drift and shift (8). The same situation may also occur with SARS-COV-2. Some studies have reported spike mutations and attempted to clarify the transmissibility change associated with new mutations $(9,10)$.

Currently, SARS-COV-2 remains a lasting threat to public health, causing mild respiratory system disease similar to that caused by seasonal influenza virus. To investigate the COVID-19 clinical progression, prognosis and SARS-COV-2 epidemic trends, we systematically contrasted the proportions or values of epidemiologic characteristics, clinical features, blood abnormalities, progressive symptoms, and hospitalization rates between influenza and COVID-19. Seasonal influenza was prevalent in winter, and COVID-19 also emerged in the last winter. The parameters of coinfection patients who presented with both COVID-19 and flu were also compared with those of COVID-19 patients. In addition, the mutants of influenza virus HA and SARS-COV-2 spike causing the epidemic were resolved and discussed. We expect that this comparative research will aid pandemic control and be beneficial to the clinical diagnosis of SARS-COV-2.

\section{MATERIALS AND METHODS}

\section{Ethics}

This study was approved by the Institutional Review Committee of Shiyan Renmin Hospital of Hubei University of Medicine, and no informed consent was required. This study was designed as a retrospective case analysis, with no patients directly involved in the study design, question setting, or outcome evaluation.

\section{Study Population}

From January 23, 2020 to February 27, 2020, the confirmed COVID-19 patients and influenza patients on outpatient visits and admission to Shiyan Renmin Hospital were uniformly collected. The clinical data of 75 COVID-19 patients, 41 influenza patients and 23 coinfection patients were retrospectively collected at the same time. All patients underwent chest computed tomography (CT) scanning at admission. Flu patients were infected with the seasonal influenza A or B virus, COVID19 patients were infected with SARS-COV-2, and coinfection patients were infected with both SARS-COV-2 and influenza $A$ or $B$ virus or parainfluenza virus (PIV). The clinical subtype for COVID-19 was screened according to the Diagnosis and Treatment Protocol for Novel Coronavirus Pneumonia (Trial Version 7) which was released by the National Health
Commission \& National Administration of Traditional Chinese Medicine (11). Influenza diagnosis and treatment plan (2019 version) (12) was used for screening the flu patients.

\section{Laboratory Examinations}

After admission to the hospital, specimens from all patients were screened for SARS-COV-2 using throat swabs. The positive result of real-time reverse transcription-polymerase chain reaction (RT-PCR) confirmed the infection. Other respiratory pathogens were detected by indirect immunofluorescent assay using IgM antibodies, including Legionella pneumophila, Coxiella burnetii, Chlamydia pneumoniae, Mycoplasma pneumoniae, adenovirus $(\mathrm{AdV})$, influenza A virus, influenza $\mathrm{B}$ virus, parainfluenza virus (PIV type $1+2+3$ ), and respiratory syncytial virus (RSV). Sputum or body fluids were also examined at admission for other possible infections with bacteria or fungi.

\section{Data Collection}

The epidemiological data, symptoms, laboratory abnormalities on admission, clinical treatments, and outcomes were recorded and collected. The examination of WBC, influenza virus antigen, joint test of nine respiratory tract pathogens, procalcitonin (PCT), hypersensitive C-reactive protein (CRP), erythrocyte sedimentation rate (ESR), blood gas analysis, and chest computed tomography (CT) were completed in the hospital. The above information was extracted from the case database of Shiyan Renmin Hospital.

\section{Sequence Alignment and Mutation Analysis}

The genome sequences of SARS-COV-2 were downloaded from the GISAID database (13). Multiple sequences were aligned using MAGE-X software (version 10.0.5), phylogenetic analysis was completed through multiple comparisons using neighbor-joining algorithms, and the number of bootstraps was 500. All spike mutations were referenced to the website of the SARS-COV-2 Sequence Analysis pipeline (14).

\section{Dynamic Analysis of HA and Spike Structure}

To analyze the dynamic model of the spike protein, we used the PDB file 6VXX. The model 1RUZ was used to validate the HA structure of influenza. PyMOL software (version 2.3.2) was used to map the S477N and N439K domain onto the 3D structure. $\mathrm{MM} / \mathrm{GBVI}$ was used to calculate the binding free energy of each conformation with receptor ACE2 (15), ACE2 (PDB ID: 1R42) was used for computation.

\section{Statistical Analysis}

SPSS 22.0 software (SPSS, Inc. Chicago, USA) was used for statistical analysis of the obtained data, and the measurement data are shown as the medians and interquartile ranges (IQRs), which were compared with the Mann-Whitney $U$-test. The categorical variables are shown as numbers (\%) and were compared with the $\chi^{2}$ test or Fisher's exact test. 
TABLE 1 | Demographic and epidemiologic characteristics of flu, COVID-19 and coinfection patients.

\begin{tabular}{lccc}
\hline & $\begin{array}{c}\text { Flu (Influenza } \\
\mathbf{A} / \mathbf{B}, \\
\boldsymbol{n}=\mathbf{4 1})\end{array}$ & $\begin{array}{c}\text { CoVID-19 } \\
(\boldsymbol{n}=\mathbf{7 5})\end{array}$ & $\begin{array}{c}\text { Coinfection } \\
\text { (Influenza A/B, } \\
\text { PIV } \boldsymbol{n}=\mathbf{2 3})\end{array}$ \\
\hline Age & $48.21 \pm 10.30$ & $47.93 \pm 10.55$ & $54.75 \pm 9.24$ \\
Gender ratio (M/F) & 1.05 & 1.27 & 1.3 \\
Incubation period (days) & $1.4(1.3,1.5)$ & $5.1(4.5,5.8)$ & $4.9(4.1,5.9)$ \\
Severity of illness & $3(7.32 \%)$ & $9(12.00 \%)$ & $5(21.74 \%)$ \\
Clear epidemiology history & $10(24.39 \%)$ & $59(78.67 \%)$ & $19(82.61 \%)$ \\
\hline
\end{tabular}

\section{RESULTS}

\section{Epidemiological and Population Characteristics of COVID-19 and Influenza Patients}

From January 23, 2020 to February 27, 2020, a total of 141 suspected patients were admitted to the isolation ward of our hospital, of which 75 were diagnosed with COVID-19 pneumonia, and 23 patients were determined to be coinfected with influenza A or B or parainfluenza virus. As shown in Table 1, the median age of COVID-19 patients was 47.93 years old, and there were 42 males and 33 females, which included 66 mild patients and 9 critically ill patients. The male/female ratio of influenza patients was 1.05, while that of COVID-19 patients was 1.27. COVID-19 patients with SARS-COV-2 infection had a longer incubation period of 5.1 days, while flu patients developed symptoms after 1.4 days. Perhaps the longer incubation period of SARS-COV-2 is adverse to the epidemiological investigations, as $18-21 \%$ of COVID-19 patients did not have a clear infection path. There may have been more asymptomatic patients among the flu patients, as only $24 \%$ of patients could be traced to an infection source. COVID-19 patients had a severe rate of $12 \%$, and coinfection patients had a higher severe proportion $(21.74 \%)$.

\section{Clinical Characteristics and Laboratory Tests of Patients in Different Groups}

The clinical characteristics of the patients are shown in Table 2. Some COVID-19 patients, flu patients and coinfection patients had the common manifestation of fever and cough. A total of 69.33 and $9.33 \%$ of COVID-19 patients had fever and nasal obstruction and rhinorrhea, respectively, which were all lower rates than those for the flu patients $(P=0.0263, P=0.0083)$. Fatigue reported in $36 \%$ of COVID-19 patients was higher than the rate in flu patients $(P=0.0023)$. Patients coinfected with SARS-COV-2 and influenza virus or PIV had a lower cough rate than COVID-19 patients $(P=0.0310)$. Other symptoms included headache, nausea, vomiting, and diarrhea. For routine blood tests, a higher proportion of flu patients developed leukopenia than COVID-19 patients (31.71 vs. $12.00 \%, P=0.0096$ ), while coinfection patients had a decreased proportion $(4.35 \%)$ of leukopenia relative to that of the COVID-19 patients, but there was no significant difference $(P=0.2889)$. A total of $70.67 \%$ of COVID-19 patients had increased CRP, sharing a median of
$13.63 \mathrm{mg} / \mathrm{L}$, which was higher than that of the flu group $(P=$ 0.0372 ). The median of an increased proportion of ESR was not significantly different among the three groups. The increase of ESR ( 48.00 vs. $53.66 \%$ ) and D-dimer (37.33 vs. $36.58 \%$ ) was a common phenomenon in the COVID-19 and flu patients. The creatinine was significantly increased in the COVID-19 group than flu group $(P=0.0239)$, while creatine kinase was elevated when there were COVID-19 patients ( $16.00 \%$ or 30.43 vs. $2.44 \%$ ).

\section{Radiological Finding and Clinical Outcome Comparison}

Among the 75 confirmed COVID-19 patients, 45 showed typical signs of viral pneumonia on chest CT. The other 23 patients showed lung infections but no typical signs on chest CT. In total, $90.67 \%$ of COVID-19 patients developed pneumonia, which is much higher than the proportion of influenza patients with pneumonia (17.07\%, $P<0.0001$ ) (Table 3), and this proportion increased to $95.65 \%$ in coinfection patients. The lesions for COVID-19 pneumonia were mostly in the subpleural area, with patchy or lumpy appearance (Figure 1A). The density of the lesions was commonly ground-glass opacities (GGOs), and there were real changes and thickened leaflet intervals. Influenza pneumonia showed GGOs with fewer solid components (Figure 1B). Twenty-one COVID-19 patients (28\%) were diagnosed with underlying diseases (Table 3), and the top three were hypertension (13.33\%), diabetes (8\%), and coronary heart disease $(5.33 \%)$. The proportion and order of underlying diseases in the flu group and coinfection group were consistent. A total of $18.67 \%$ of COVID-19 patients developed complications of liver injury, which was a higher rate than that of flu patients (4.88\%, $P=0.0395)$. No flu patient had a complication of acute respiratory distress syndrome (ARDS), with a proportion of $12 \%$ in the COVID-19 group $(P=0.0209)$. In the coinfection group, ARDS was the most common complication (17.39\%), followed by liver injury (13.04\%) and kidney injury (8.70\%).

\section{Hospitalization and Treatment for Patients in Different Groups}

The median hospitalization period of COVID-19 patients was 19 days, while flu patients after admission required only 4 days to discharge $(P<0.0001)$ (Table 4$)$. According to the Diagnosis and Treatment Protocol for COVID-19 (11), all COVID-19 patients received broad-spectrum antiviral treatment, including interferon- $\alpha$ sprays, arbidol hydrochloride, or lopinavir and ritonavir (16). Antibiotics, including cephalosporins, carbapenem, quinolones and so on, and antifungal drugs were used when appropriate. By the end of the study period, all patients were being treated in the hospital, and $98.67 \%$ of COVID-19 patients were cured and survived; only one patient died (1.33\%). All flu and coinfection patients were cured and discharged.

\section{Pathogen Comparisons for Influenza Virus, SARS-COV and SARS-COV-2}

Influenza virus belongs to the family Orthomyxoviridae, whose genome contains eight RNA segments. SARS-COV and 
TABLE 2 | Clinical characteristics and selected laboratory abnormalities of flu, COVID-19 and coinfection patients.

Influenza $(n=41)$
COVID-19 $(n=75)^{\mathrm{a}}$

\begin{tabular}{lc} 
Fever $\left(\geq 37.3^{\circ} \mathrm{C}\right)$ & $36(87.80 \%)$ \\
Cough & $21(51.22 \%)$ \\
Nasal obstruction and rhinorrhea & $12(29.27 \%)$ \\
Sore throat & $4(9.76 \%)$ \\
Shortness of breath and chest tightness & $3(7.32 \%)$ \\
Fatigue & $4(9.76 \%)$ \\
Diarrhea and vomiting & $3(7.32 \%)$ \\
Blood routine & \\
WBC count $\left(\times 10^{9} / \mathrm{L}\right)$ & $5.18(3.71,8.12)$ \\
$\left(\leq 3.5 \times 10^{9} / \mathrm{L}\right)$ & $13(31.71 \%)$ \\
Lymphocyte count $\left(\times 10^{9} / \mathrm{L}\right)$ & $1.20(0.83,1.62)$ \\
$(\leq 1.1 \times 109 / \mathrm{L})$ & $15(36.59 \%)$ \\
CRP $(\mathrm{mg} / \mathrm{L})$ & $7.43(4.26,17.40)$ \\
( $\geq 5 \mathrm{mg} / \mathrm{L})$ & $21(51.22 \%)$ \\
ESR(mm/h) & $18(7.50,33.50)$ \\
( $\geq 15 \mathrm{~mm} / \mathrm{h})$ & $22(53.66 \%)$ \\
(D-dimer mg/L) & $0.17(0.10,0.28)$ \\
( $\geq 0.25 \mathrm{mg} / \mathrm{L})$ & $15(36.58 \%)$ \\
Creatine $\mathrm{kinase}(\mathrm{U} / \mathrm{L})$ & $104(66.30,149.60)$ \\
$(\geq 171 \mathrm{U} / \mathrm{L})$ & $1(2.44 \%)$ \\
BUN $(\mathrm{mmol} / \mathrm{L})$ & $4.43(3.69,5.26)$ \\
Creatinine $(\mu \mathrm{mol} / \mathrm{L})$ & $86.32(78.90,92.90)$ \\
( $\geq 104 \mu \mathrm{mol} / \mathrm{L})$ & $3(7.32 \%)$ \\
\hline
\end{tabular}

\section{Coinfection (Influenza} $A / B, n=23)^{b}$

\section{Clinical characteristics}

$52(69.33 \%)$
$49(65.33 \%)$
$7(9.33 \%)$
$14(18.67 \%)$
$9(12.00 \%)$
$27(36.00 \%)$
$7(9.33 \%)$
$5.52(4.19,7.24)$
$9(12.00 \%)$
$1.23(0.90,1.59)$
$20(26.67 \%)$
$13.63(3.93,26.60)$
$53(70.67 \%)$
$17(7.00,31.50)$
$36(48.00 \%)$
$0.25(0.14,0.34)$
$28(37.33 \%)$
$118(78.50,158.30)$
$12(16 \%)$
$4.38(3.52,5.21)$
$90.94(74.70,108.10)$
$18(24 \%)$

$$
\begin{gathered}
15(65.22 \%) \\
9(39.13 \%) \\
5(21.74 \%) \\
4(17.39 \%) \\
2(8.70 \%) \\
6(26.09 \%) \\
2(8.70 \%)
\end{gathered}
$$
$5.34(4.51,6.23)$
1 (4.35\%)
$1.39(1.05,1.76)$
$5(21.74 \%)$

$14.82(4.87,28.41)$

$14(60.87 \%)$

$21.5(9.50,41.00)$

$12(52.17 \%)$

$0.28(0.18,0.37)$

$12(52.17 \%)$

$115(62.40,175.20)$

$7(30.43 \%)$

$4.50(3.76,5.03)$

$97.99(76.70,118.04)$

6 (26.09\%)

\begin{tabular}{|c|c|c|c|c|}
\hline & Influenza ( $n=41)$ & COVID-19 $(n=75)^{\mathrm{a}}$ & $\begin{array}{l}\text { Coinfection (Influenza } \\
\text { A/B, } n=23)^{\mathrm{b}}\end{array}$ & $P$ value ${ }^{\mathrm{a}, \mathrm{b}}$ \\
\hline Underlying diseases & $11(26.83 \%)$ & $21(28.00 \%)$ & $7(30.43 \%)$ & $1.0000,0.7981$ \\
\hline Diabetes & $3(7.32 \%)$ & $6(8.00 \%)$ & $2(8.70 \%)$ & $1.0000,1.0000$ \\
\hline Coronary heart disease & $3(7.32 \%)$ & $4(5.33 \%)$ & $1(4.35 \%)$ & $0.6965,1.0000$ \\
\hline Acute respiratory distress syndrome & 0 & $9(12.00 \%)$ & $4(17.39 \%)$ & $0.0209,0.5049$ \\
\hline Shock & 0 & $1(1.33 \%)$ & 0 & $1.0000,1.0000$ \\
\hline Liver injury & $2(4.88 \%)$ & $14(18.67 \%)$ & $3(13.04 \%)$ & $0.0395,0.5333$ \\
\hline Kidney injury & 0 & 6 (8.00\%) & 2 (8.70\%) & $0.0629,0.9151$ \\
\hline
\end{tabular}

$\boldsymbol{P}_{\text {value }}^{\mathrm{a}, \mathrm{b}}$

$0.0263,0.7989$
$0.1662,0.0310$
$0.0083,0.1454$
$0.2855,1.0000$
$0.5353,1.0000$
$0.0023,0.3788$
$1.0000,1.0000$

$0.2112,0.6358$
$0.0096,0.2889$
$0.9678,0.0627$
$0.2659,0.6353$
$0.0617,0.2026$
$0.0372,0.3768$
$0.4271,0.5078$
$0.6979,0.7261$
$0.0046,0.1246$
$0.4311,0.0018$
$0.1157,0.8316$
$0.0013,0.0027$
$0.9550,0.5377$
$0.0008,0.1659$
$0.0239,0.5840$

The data was shown as $n$ (\%) or median (IQR).

${ }^{a}$ COVID-19 vs. Influenza group.

${ }^{b}$ Coinfection vs. COVID-19 group.

TABLE 3 | Underlying diseases and progressive symptoms of flu, COVID-19 and coinfection patients.

The data was shown as $n$ (\%).

${ }^{a}$ COVID-19 vs. Influenza group.

${ }^{b}$ Coinfection vs. COVID-19 group.

SARS-COV-2 belong to the $\beta$-coronavirus (CoV) genus in the Coronaviridae family (17). SARS-COV-2 showed a high nucleotide sequence identity (79.5\%) with SARS-COV (18). Recent reports have shown that SARS-COV-2 enters susceptible cells through binding with the receptor angiotensin-converting enzyme 2 (ACE2), which is the same as SARS-COV (19-21). All ages of people are susceptible to influenza A virus (22), while SARS-COV and SARS-COV-2 primarily infect adults (23). 
A

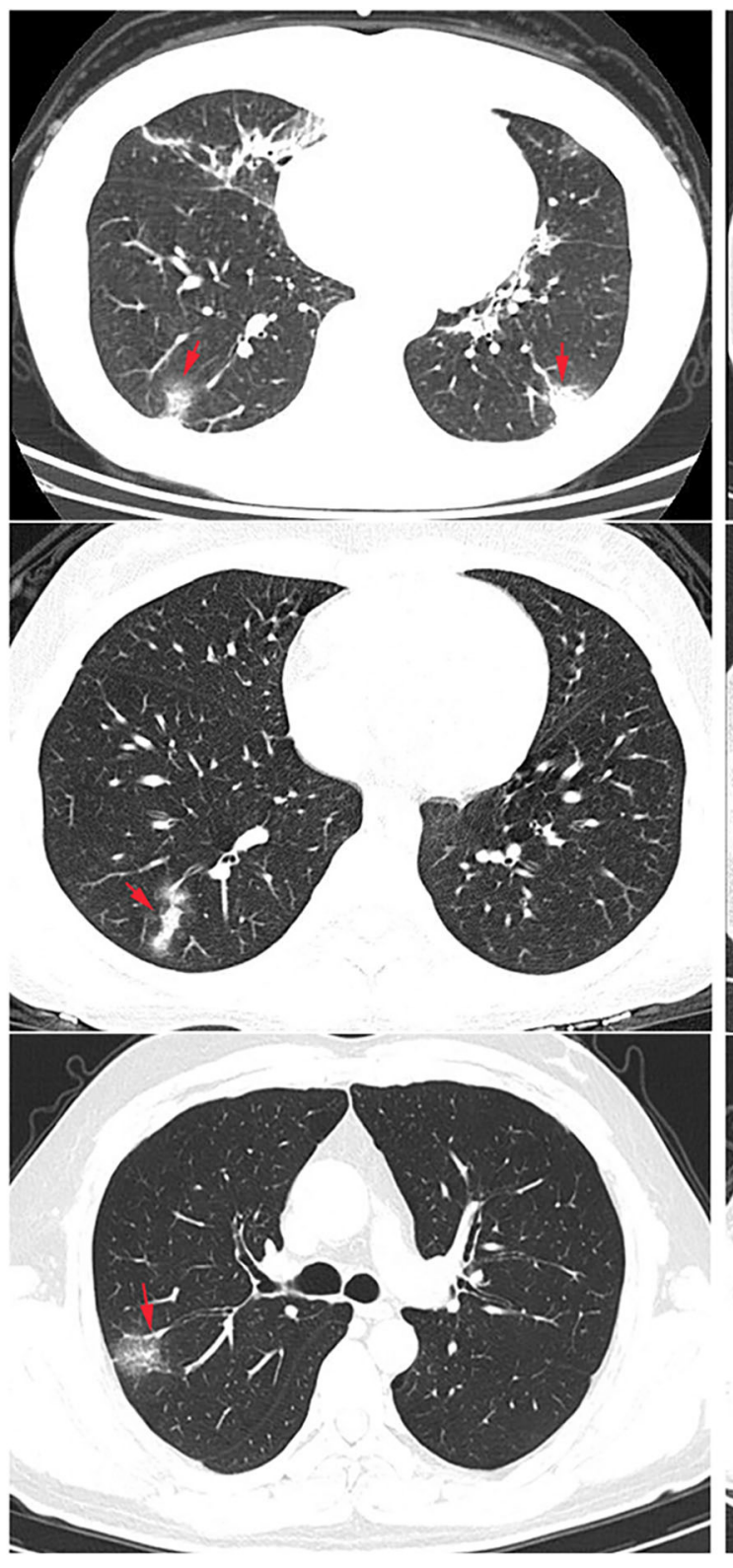

B

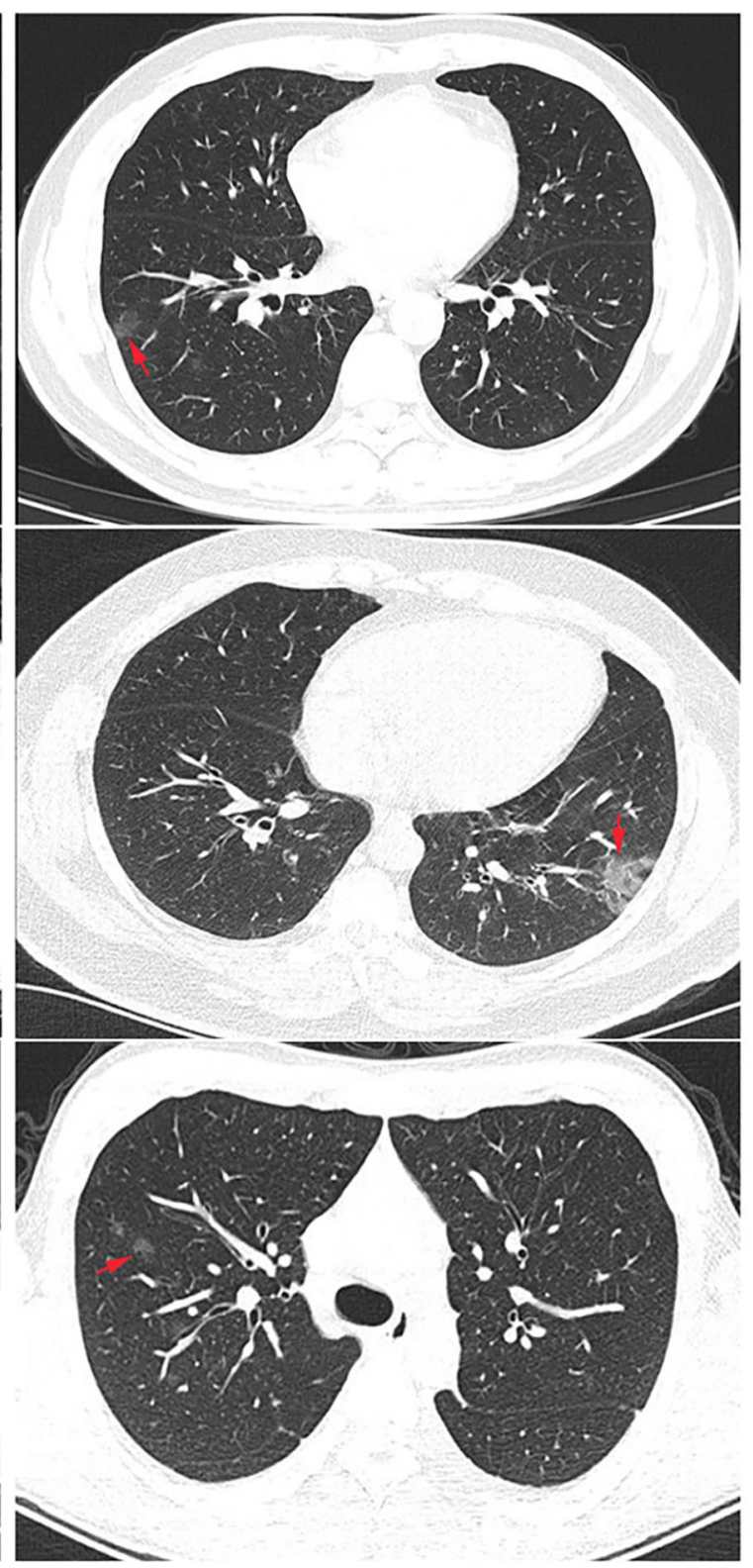

FIGURE 1 | Radiological findings: chest computer tomography (CT) images of COVID-19 and flu pneumonia at the same time after onset. (A) COVID-19 pneumonia showed multiple ground glass opacity with solid components in the bilateral subpleural area. The nucleic acid test for SARS-CoV-2 was positive. Arrows showed the lesions. (B) CT examination showed scattered ground glass opacity of both lungs in an influenza patient, mainly in the lung periphery with less solid components. SARS-CoV-2 nucleic acid test was negative for three times, and immunofluorescence test was positive for influenza A virus.

Because all three viruses can bind with receptors in the upper respiratory tract of humans, they are all easily transmitted by airborne droplets during coughing, sneezing or intimate conversation. The reproductive number $\left(\mathrm{R}_{0}\right)$ is defined as the average number of secondary cases generated per confirmed infectious case. The reported median $\mathrm{R}_{0}$ for seasonal influenza virus is 1.28 (IQR: 1.19-1.37), except for during the 2009 pandemic (Table 5) (24). SARS-COV-2 had a median $\mathrm{R}_{0}$ of 2.38 at the beginning of the epidemic (25), which is still controversial, but it is probably higher than the $\mathrm{R}_{0}$ of SARS-COV (1.7-1.9) (26). Surveillance of genome mutation dynamics is critical for the effective control of diseases. To date, SARS-COV-2 seems to exhibit a higher mutation rate than influenza virus per site per year $\left[(1.12-6.25) \times 10^{-3}\right.$ vs. $\left.(0.60-2.00) \times 10^{-6}\right](27-29)$, which remains to be further determined. Relatively, SARS-COV has a similar evolution rate $\left(0.80-2.38 \times 10^{-3}\right.$ per site per year) (30). 
TABLE 4 | Hospitalization for flu, COVID-19 and coinfection patients.

\begin{tabular}{|c|c|c|c|c|}
\hline & Influenza $(n=41)$ & COVID-19 $(n=75)^{\mathrm{a}}$ & $\begin{array}{l}\text { Coinfection (Influenza } \\
\qquad A / B, n=23)^{b}\end{array}$ & $P$ value $^{\mathrm{a}, \mathrm{b}}$ \\
\hline Hospitalization period (days) & $3.95(3,5)$ & $19.12(11,26)$ & $19.48(13,25)$ & $<0.0001,0.5424$ \\
\hline Treatment & Oseltamivir, Peramivir & Following the guideline & $\begin{array}{c}\text { Oseltamivir, Peramivir (for } \\
\text { influenza A/B) }\end{array}$ & \\
\hline Cure rate & 41 (100\%) & $74(98.67 \%)$ & $23(100 \%)$ & $0.4577,0.5778$ \\
\hline Fatality rate & 0 & $1(1.33 \%)$ & 0 & $0.4577,0.5778$ \\
\hline
\end{tabular}

The data was shown as $n$ (\%).

${ }^{a}$ COVID-19 vs. Influenza group.

${ }^{b}$ Coinfection vs. COVID-19 group.

TABLE 5 | Pathogen comparisons for seasonal flu virus, SARS-COV-2 and SARS-COV.

\begin{tabular}{lccc}
\hline & $\begin{array}{c}\text { Seasonal } \\
\text { influenza virus } \\
\text { (H1N1, H3N2) }\end{array}$ & SARS-COV-2 & SARS-COV \\
\hline Family & Orthomyxoviridae & Coronaviridae & Coronaviridae \\
Susceptible crowd & Children and & Children and & Adults \\
Transmission & adults & adults & Droplets \\
Ro & Droplets & Droplets & $1.7-1.9$ \\
Mutation (/site/year) & $0.60-2.00 \times 10^{-6}$ & $1.12-6.25 \times 10^{-3}$ & $0.80-2.38 \times 10^{-3}$
\end{tabular}

\section{Adaptive Mutations of Influenza Virus and SARS-COV-2}

Haemagglutinin (HA) on influenza virus and spike on SARSCOV-2 are both responsible for binding to the receptors on permissive cells. HA and spike are both homotrimers, where each monomer comprising two subunits, HA1 and HA2 or S1 and S2 (Figures 2A,B). Several important adaptive mutations occur in HA, including E190D and G225D for H1N1 and Q226L and G228S for H2N2 and H3N2 (31). Some mutations in the polymerase subunits $\mathrm{PB} 1, \mathrm{~PB} 2$, and PA are critical for increasing polymerase activity and viral virulence (32). As of 2 June 2020, more than ten thousand mutant sequences of SARS-COV-2 were uploaded to the GISAID database (13). There were 18,539 nonsynonymous mutations on the spike protein, the D614G mutation was unusually enriched and present in more than 8,000 strains. Up to February 1 st 2021, nearly 300,000 strains containing D614G variation, A222V and L18F were the second and third mutations (Figure 2C). N439K, S477N and N501Y which located in the receptor-binding domain may affect the immunogenicity or vaccination. Our result showed S477N and N439K mutations have the capability to enhance the affinity with receptor (Figures 2D,E). Additional studies are needed to elucidate the effect of mutations in which are not located in the receptor-binding domain (pocket) on influenza virus and SARS-COV-2 infection and their epidemiological outcomes.

\section{DISCUSSION}

To date, most epidemiological reports have clarified the case disparities in clinical manifestations, routine blood tests, and immunity factors of COVID-19 (33-35). However, the dual epidemics of COVID-19 and influenza makes the diagnosis, treatment, and vaccination face greater challenges, even though some studies have assessed the differences between influenza and COVID-19 in terms of clinical characteristics or outcomes $(36,37)$. Both are respiratory virus infections, and influenza and COVID-19 have many of the same symptoms, including respiratory system and gastrointestinal system symptoms. Severe cases also have loss of taste or smell, difficulty breathing, or shortness of breath (38). COVID-19 may cause gastrointestinal problems, such as diarrhea, vomiting, and abdominal pain (39). Both the SARS-COV-2 receptor ACE2 and cellular serine protease transmembrane protease serine 2 (TMPRSS2) are critical for the fusion of viral and cellular membranes, which are not only expressed in lung alveolar type 2 cells and gland cells but also highly expressed in the ileum and colon $(40,41)$, suggesting that the virus can invade the digestive tract and intestine, and viral RNA can be detected in patients' stool.

Certain risk factors predispose patients to increased morbidity and mortality following exposure to the influenza A virus or SARS-COV-2. Age is the most significant risk factor for influenza and COVID-19-related mortality $(42,43)$. Age may also have different effects on the results of influenza virus infection between males and females (44). For COVID-19, the sex difference is a significant factor for mortality. Recently reported data showed that the male mortality rate is 2.4 times that of females (70.3 vs. $29.7 \%$ ) (43), and there were 1641 men among the 2248 confirmed COVID-19 patients, which was 2.6 times the proportion of women (73 vs. 27\%) (45). Chronic respiratory diseases and cardiovascular diseases are the two major comorbidities for influenza patients (5), and other comorbidities associated with poor influenza outcomes include diabetes and obesity. In our study, the most common underlying diseases in both flu and COVID-19 patients were hypertension, diabetes, and coronary heart disease. There was a significant difference in the progressive symptoms and complications of pneumonia between influenza and COVID-19 illness, including concurrency and CT radiology features. Only $17 \%$ of flu patients developed pneumonia, while the number increased to $90.67 \%$ in COVID-19 patients. In the 


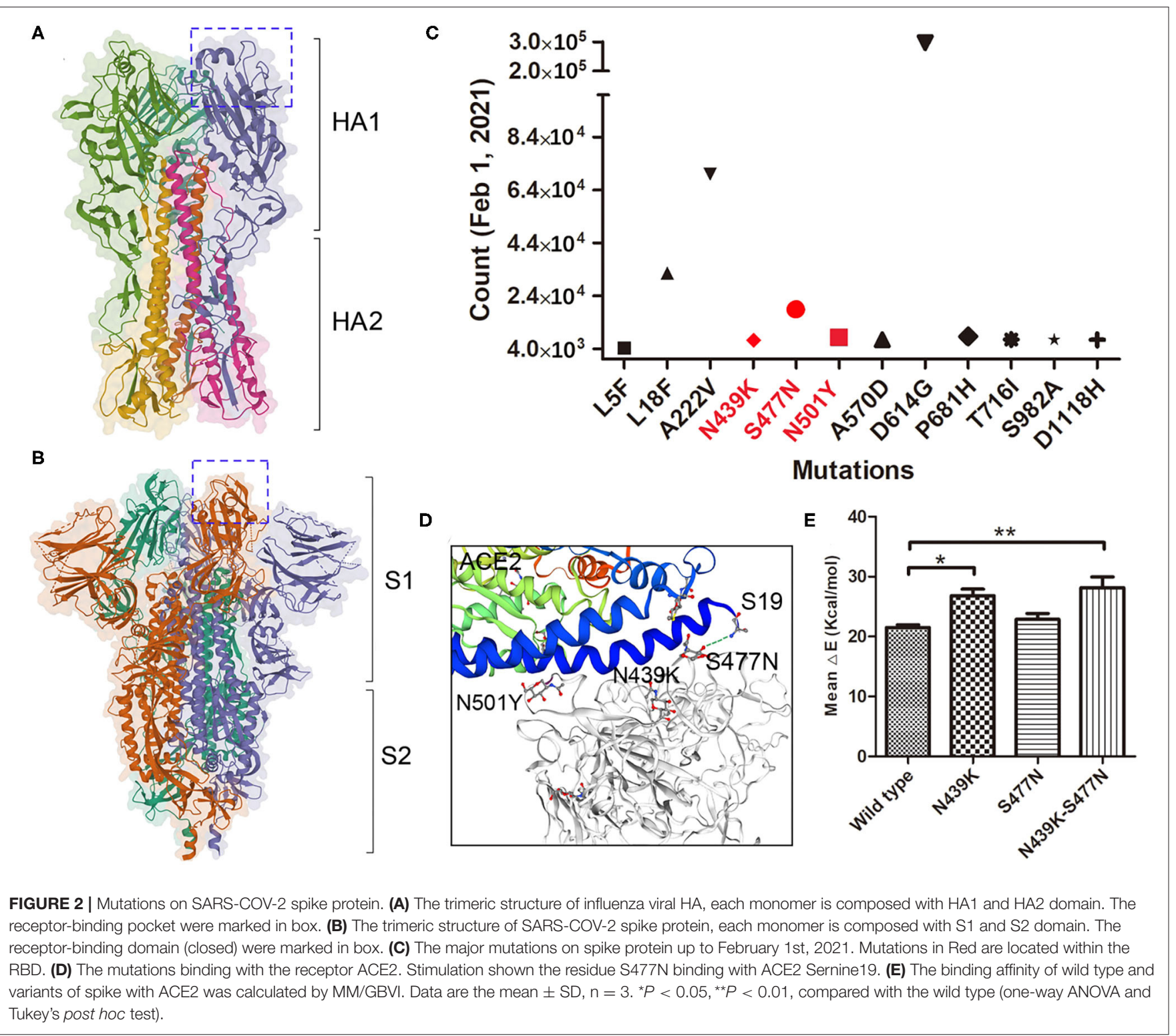

early stage of flu pneumonia, X-ray imaging showed thickened and blurred lung texture and small patchy shadows. The advanced stage (onset 3-7 days) is dominated by GGOs and consolidation (46). COVID-19 pneumonia shows limited patchy shadows in the early stage, diffuse lung abnormalities, and multiple consolidations when the lesions are severe (47).

Dynamic changes in routine blood parameters refer to the evaluation of treatment or examination of the disease by observing the quantity change and shape distribution in blood cells. The total count of WBCs, neutrophils, lymphocytes, and monocytes progressively decreased, which may be related to the direct invasion of the virus into hematopoietic cells (48). The initial CRP of severe COVID-19 patients increased prior to CT findings (49), and the CRP value increased rapidly after admission, indicating a strong inflammatory response; the virus is prevalent in patients' bodies at this stage. ESR, elevated by the acute-phase response, can be used as an important indicator to distinguish patients with severe COVID-19 in the early stage (50). Severe COVID-19 illness is associated with a prominent elevation in ESR, which may provide additional information on disease progression. The BUN and blood creatinine levels rise rapidly before death in severe COVID-19 patients, this is consistent with our study (51). The inadequate sample size may limit the conclusion of our study, further studies are needed to analyze the dynamic changes in immune factors for COVID-19 progression and prognosis.

Several adaptation mutations have been identified in different segments of influenza A virus, and thoroughly researched mutations, including E627K and D701N on PB2 of avian flu virus and other subtypes, promote polymerase activity and adaption to act cooperatively with humans $(52,53)$. Adaptive mutations in SARS-COV-2 have also been reported, and the 
most important mutation in spike is D614G, which has attracted global attention $(54,55)$. The D614G mutant began to spread in Europe in early February 2020 and became the mainstream strain worldwide by May 2020, accounting for nearly $70 \%$ of samples in Europe and North America (56). Zhang et al. (57) latest research showed that small genetic mutations in the SARS-COV2 variants are prevalent throughout Europe and the United States, which may increase the number of spike proteins on virions, and it will greatly improve the viral infectivity by 9-10 times. The N439K variants can maintain fitness but can evade the antibody-mediated immunity (58), it is unclear what the S477N mutation will bring to the vaccines and epidemics. More studies are needed to compare the clinical outcomes and prevalence between adaptive mutants and wild type.

This study provides new insight into the differences in clinical outcomes, laboratory abnormalities, comorbidities and hospitalization between influenza and COVID-19 patients and discusses the relationship between viral adaptive mutations and protein function, which will provide a reference for clinical differential diagnosis and epidemic surveillance.

\section{DATA AVAILABILITY STATEMENT}

The original contributions presented in the study are included in the article/supplementary material, further inquiries can be directed to the corresponding author/s.

\section{ETHICS STATEMENT}

Written informed consent was obtained from the individual(s) for the publication of any potentially identifiable images or data included in this article.

\section{AUTHOR'S NOTE}

COVID-19 remains one of the key threats to public health. SARS-COV-2 causes a mild respiratory system disease, which has symptoms similar to those of seasonal influenza virus. COVID-19

\section{REFERENCES}

1. Coronaviridae Study Group of the International Committee on Taxonomy of V. The species Severe acute respiratory syndrome-related coronavirus: classifying 2019-nCoV and naming it SARS-CoV-2. Nat Microbiol. (2020) 5:536-44. doi: 10.1038/s41564-020-0695-Z

2. Li Q, Guan X, Wu P, Wang X, Zhou L, Tong Y, et al. Early transmission dynamics in Wuhan, China, of novel coronavirus-infected pneumonia. N Engl J Med. (2020) 382:1199-207. doi: 10.1056/NEJMoa2001316

3. University JH. COVID-19 Dashboard by the Center for Systems Science and Engineering (CSSE) at Johns Hopkins University. (2020). Available online at: https://www.arcgis.com/apps/opsdashboard/index.html\#/ bda7594740fd40299423467b48e9ecf6

4. Dhama K, Sharun K, Tiwari R, Dadar M, Malik YS, Singh KP, et al. COVID-19, an emerging coronavirus infection: advances and prospects in designing and developing vaccines, immunotherapeutics, and therapeutics. Hum Vaccin Immunother. (2020) 16:1232-8. doi: 10.1080/21645515.2020. 1735227

5. Organization WH. Sex, Gender and Influenza. WHO (2010). and seasonal influenza both emerged in the respiratory virus season. Comparison of clinical features and outcomes of medically attended COVID-19 and influenza A patients will help us to better diagnose and treat the infection caused by SARS-COV-2. In this study, we systematically contrasted the proportions or values of epidemiologic characteristics, clinical features, blood abnormalities, progressive symptoms, and hospitalization between influenza and COVID-19. The parameters of coinfection patients who presented with both COVID-19 and flu were also compared with the COVID-19 cases. In addition, mutants of influenza virus HA and SARSCOV-2 spike causing the epidemic were resolved and discussed according to the literature or computation. This comparative research aims to aid pandemic control and be beneficial to clinical diagnosis.

\section{AUTHOR CONTRIBUTIONS}

LL, FZ, JY, and ZxL contributed to the design of experiments. LL, FZ, JR, SY, MJ, XuL, ZjL, XiL, WD, YL, and HT contributed to the conduction of experiments. LL, FZ, JR, SY, MJ, and ZjL contributed to the reagents. LL, FZ, JR, SY, MJ, XuL, ZjL, XiL, JL, $\mathrm{JZ}$, and $\mathrm{ZxL}$ contributed to the analyses of the data. $\mathrm{LL}$ and $\mathrm{ZxL}$ contributed to the writing the paper. JY and ZxL contributed to the editing the paper. All authors contributed to the article and approved the submitted version.

\section{FUNDING}

This work was supported by Natural Science Foundation of China (82002149 and81902066), the Natural Science Foundation of Hubei Provincial Department of Education (Q20192105 and Q20192104), the Foundation of Health Commission of Hubei Province (WJ2021M059), and the COVID-19 Epidemic Emergency Research Foundation (2020XGFYZR07 and 2020XGFYZR08). The funder has no function in the study design, data collection and analysis, manuscript writing and submission.

6. Franco-Paredes C, Hernandez-Ramos I, Del Rio C, Alexander KT, TapiaConyer R, Santos-Preciado JI. H1N1 influenza pandemics: comparing the events of 2009 in Mexico with those of 1976 and 1918-1919. Arch Med Res. (2009) 40:669-72. doi: 10.1016/j.arcmed.2009.10.004

7. Chuah CXP, Lim RL, Chen MIC. Investigating the Legacy of the 1918 influenza pandemic in age-related seroepidemiology and immune responses to subsequent influenza $\mathrm{A}(\mathrm{H} 1 \mathrm{~N} 1)$ viruses through a structural equation model. Am J Epidemiol. (2018) 187:2530-40. doi: 10.1093/aje/ kwy192

8. Kim H, Webster RG, Webby RJ. Influenza virus: dealing with a drifting and shifting pathogen. Viral Immunol. (2018) 31:174-83. doi: 10.1089/vim.2017.0141

9. Laha S, Chakraborty J, Das S, Manna SK, Biswas S, Chatterjee R. Characterizations of SARS-CoV-2 mutational profile, spike protein stability and viral transmission. Infect Genet Evol. (2020) 85:104445. doi: 10.1016/j.meegid.2020.104445

10. Lau SY, Wang P, Mok BW, Zhang AJ, Chu H, Lee AC, et al. Attenuated SARSCoV-2 variants with deletions at the S1/S2 junction. Emerg Microbes Infect. (2020) 9:837-42. doi: 10.1080/22221751.2020.1756700 
11. Zhao JY, Yan JY, Qu JM. Interpretations of "Diagnosis and treatment protocol for novel coronavirus pneumonia (trial version 7)". Chin Med J. (2020) 133:1347-9. doi: 10.1097/CM9.0000000000000866

12. Health, NHCOO. Influenza Diagnosis and Treatment Plan (2019 Version). (2019). Available online at: http://www.nhc.gov.cn/ yzygj/s7653p/201911/a577415af4e5449cb30ecc6511e369c7/files/ 75a810713dc14dcd9e6db8b654bdef79.pdf

13. GISAID. Global Initiative of Sharing All Influenza Data. (2020). Available online at: https://www.gisaid.org

14. Group TSCG. COVID-19 Viral Genome Analysis Pipeline [Online]. (2020). Available online at: https://cov.lanl.gov/content/index

15. Xue X, Shi J, Xu H. Dynamics of binding ability prediction between spike protein and human ACE2 reveals the adaptive strategy of SARS-CoV-2 in humans. Sci Rep. (2021) 11:3187. doi: 10.1038/s41598-021-82938-2

16. Health NHCOO. DiagnosisandTreatmentProtocolforNovelCoronavirus Pneumonia(6rdInterim Edition) [Online]. (2020). Available online at: http:// www.gov.cn/zhengce/zhengceku/2020-02/19/content_5480948.htm

17. Pal M, Berhanu G, Desalegn C, Kandi V. Severe acute respiratory syndrome coronavirus-2 (SARS-CoV-2): an update. Cureus. (2020) 12:e7423. doi: 10.7759/cureus.7423

18. Lu R, Zhao $\mathrm{X}$, Li J, Niu P, Yang B, Wu $\mathrm{H}$, et al. Genomic characterisation and epidemiology of 2019 novel coronavirus: implications for virus origins and receptor binding. Lancet. (2020) 395:565-74. doi: 10.1016/S0140-6736(20)30251-8

19. Wang Q, Zhang Y, Wu L, Niu S, Song C, Zhang Z, et al. Structural and functional basis of SARS-CoV-2 entry by using human ACE2. Cell. (2020) 181:894-904.e899. doi: 10.1016/j.cell.2020.03.045

20. Yan R, Zhang Y, Li Y, Xia L, Guo Y, Zhou Q. Structural basis for the recognition of SARS-CoV-2 by full-length human ACE2. Science. (2020) 367:1444-8. doi: 10.1126/science.abb2762

21. Zhou P, Yang XL, Wang XG, Hu B, Zhang L, Zhang W, et al. A pneumonia outbreak associated with a new coronavirus of probable bat origin. Nature. (2020) 579:270-3. doi: 10.1038/s41586-020-2012-7

22. Coates BM, Staricha KL, Wiese KM, Ridge KM. Influenza A virus infection, innate immunity, and childhood. JAMA Pediatr. (2015) 169:95663. doi: 10.1001/jamapediatrics.2015.1387

23. Rabaan AA, Al-Ahmed SH, Haque S, Sah R, Tiwari R, Malik YS, et al. SARSCoV-2, SARS-CoV, and MERS-COV: a comparative overview. Infez Med. (2020) 28:174-84.

24. Biggerstaff M, Cauchemez S, Reed C, Gambhir M, Finelli L. Estimates of the reproduction number for seasonal, pandemic, and zoonotic influenza: a systematic review of the literature. BMC Infect Dis. (2014) 14:480. doi: 10.1186/1471-2334-14-480

25. Li R, Pei S, Chen B, Song Y, Zhang T, Yang W, et al. Substantial undocumented infection facilitates the rapid dissemination of novel coronavirus (SARS-CoV2). Science. (2020) 368:489-93. doi: 10.1126/science.abb3221

26. Petrosillo N, Viceconte G, Ergonul O, Ippolito G, Petersen E. COVID-19, SARS and MERS: are they closely related? Clin Microbiol Infect. (2020) 26:729-34. doi: 10.1016/j.cmi.2020.03.026

27. Zhao Z, Li H, Wu X, Zhong Y, Zhang K, Zhang YP, et al. Moderate mutation rate in the SARS coronavirus genome and its implications. BMC Evol Biol. (2004) 4:21. doi: 10.1186/1471-2148-4-21

28. Jia Y, Shen G, Zhang Y, Huang KS, Ho Y, Hor WS, et al. Analysis of the mutation dynamics of SARS-CoV-2 reveals the spread history and emergence of RBD mutant with lower ACE2 binding affinity. bioRxiv. (2020). doi: 10.1101/2020.04.09.034942

29. Takahiko Koyama DP. Variant analysis of SARS-CoV-2 genomes. Bull World Health Organ. (2020) 98:495-504. doi: 10.2471/BLT.20.253591

30. Nobusawa E, Sato K. Comparison of the mutation rates of human influenza A and B viruses. J Virol. (2006) 80:3675-8. doi: 10.1128/JVI.80.7.3675-36 78.2006

31. Lazniewski M, Dawson WK, Szczepinska T, Plewczynski D. The structural variability of the influenza A hemagglutinin receptor-binding site. Brief Funct Genomics. (2018) 17:415-27. doi: 10.1093/bfgp/elx04

32. Taft AS, Ozawa M, Fitch A, Depasse JV, Halfmann PJ, Hill-Batorski L, et al. Identification of mammalian-adapting mutations in the polymerase complex of an avian H5N1 influenza virus. Nat Commun. (2015) 6:7491. doi: 10.1038/ncomms8491
33. Puljiz I, Kuzman I, Dakovic-Rode O, Schonwald N, Mise B. Chlamydia pneumoniae and Mycoplasma pneumoniae pneumonia: comparison of clinical, epidemiological characteristics and laboratory profiles. Epidemiol Infect. (2006) 134:548-55. doi: 10.1017/S0950268805005522

34. Guan WJ, Ni ZY, Hu Y, Liang WH, Ou CQ, He JX, et al. Clinical characteristics of coronavirus disease 2019 in China. N Engl J Med. (2020) 382:170820. doi: $10.1056 /$ NEJMoa2002032

35. Xu XW, Wu XX, Jiang XG, Xu KJ, Ying LJ, Ma CL, et al. Clinical findings in a group of patients infected with the 2019 novel coronavirus (SARSCov-2) outside of Wuhan, China: retrospective case series. BMJ. (2020) 368:m606. doi: 10.1136/bmj.m606

36. Song X, Delaney M, Shah RK, Campos JM, Wessel DL, DeBiasi RL, et al. Comparison of clinical features of COVID-19 vs seasonal influenza A and B in US children. JAMA Netw Open. (2020) 3:e2020495. doi: 10.1001/jamanetworkopen.2020.20495

37. Liu M, Zeng W, Wen Y, Zheng Y, Lv F, Xiao. K. COVID-19 pneumonia: CT findings of 122 patients and differentiation from influenza pneumonia. Eur Radiol. (2020) 30:5463-9. doi: 10.1007/s00330-020-06928-0

38. Prevention CfDCA. Similarities and Differences between Flu and COVID19. (2020). Available online at: https://www.cdc.gov/flu/symptoms/flu-vscovid19.htm

39. Ng SC, Tilg H. COVID-19 and the gastrointestinal tract: more than meets the eye. Gut. (2020) 69:973-4. doi: 10.1136/gutjnl-2020-321195

40. Bilinska K, Jakubowska P, Von Bartheld CS, Butowt R. Expression of the SARS-CoV-2 entry proteins, ACE2 and TMPRSS2, in cells of the olfactory epithelium: identification of cell types and trends with age. ACS Chem Neurosci. (2020) 11:1555-62. doi: 10.1021/acschemneuro.0c00210

41. Lukassen S, Chua RL, Trefzer T, Kahn NC, Schneider MA, Muley $\mathrm{T}$, et al. SARS-CoV-2 receptor ACE2 and TMPRSS2 are primarily expressed in bronchial transient secretory cells. EMBO J. (2020) 39:e105114. doi: 10.15252/embj.20105114

42. Thompson WW, Shay DK, Weintraub E, Brammer L, Cox N, Anderson LJ, et al. Mortality associated with influenza and respiratory syncytial virus in the United States. JAMA. (2003) 289:179-86. doi: 10.1001/jama.289.2.179

43. Jin JM, Bai P, He W, Wu F, Liu XF, Han DM, et al. Gender differences in patients with COVID-19: focus on severity and mortality. Front Public Health. (2020) 8:152. doi: 10.3389/fpubh.2020.00152

44. Thomas MR, Marston L, Rafferty GF, Calvert S, Marlow N, Peacock JL, et al. Respiratory function of very prematurely born infants at follow up: influence of sex. Arch Dis Child Fetal Neonatal Ed. (2006) 91:F197201. doi: 10.1136/adc.2005.081927

45. ICNARC. ICNARC Report on COVID-19 in Critical Care. (2020). Available online at: https://www.icnarc.org/About/Latest-News/2020/04/04/ReportOn-2249-Patients-Critically-Ill-With-Covid-19

46. $\mathrm{Wu} J$, Lu AD, Zhang LP, Zuo YX, Jia YP. Zhonghua xue ye xue za zhi =. Zhonghua Xueyexue Zazhi. (2019) 40:527. doi: 10.3760/cma.j.issn.0253-2727.2019.01.010

47. Committee CotIDRGoC.S.o.R., Infectious, o.t.I.D.R.S.o.C.M.D.A., Disease Imaging Group IDB, Chinese Research Hospital Association; Imaging,; Infectious Diseases Group, et al. (Infectious, C.o.C.A.f.t.P.a.T.o.S.A.I., Disease) Guideline for imaging diagnosis of novel coronavirus (2019ncov) infected pneumonia (1st edition 2020). New Med. (2020) 30:2234. doi: 10.12173/j.issn.1004-5511.2020.01.07

48. Lu G, Wang J. Dynamic changes in routine blood parameters of a severe COVID-19 case. Clin Chim Acta. (2020) 508:98102. doi: 10.1016/j.cca.2020.04.034

49. Tan C, Huang Y, Shi F, Tan K, Ma Q, Chen Y, et al. C-reactive protein correlates with computed tomographic findings and predicts severe COVID-19 early. $J$ Med Virol. (2020) 92:856-62. doi: 10.1002/jmv.25871

50. Lapic I, Rogic D, Plebani M. Erythrocyte sedimentation rate is associated with severe coronavirus disease 2019 (COVID-19): a pooled analysis. Clin Chem Lab Med. (2020) 58:1146-8. doi: 10.1515/cclm-2020-0620

51. Wang D, Hu B, Hu C, Zhu F, Liu X, Zhang J, et al. Clinical characteristics of 138 hospitalized patients with 2019 novel coronavirus-infected pneumonia in Wuhan, China. JAMA. (2020) 323:1061-9. doi: 10.1001/jama.20 20.1585

52. Arai Y, Kawashita N, Hotta K, Hoang PVM, Nguyen HLK, Nguyen $\mathrm{TC}$, et al. Multiple polymerase gene mutations for human adaptation 
occurring in Asian H5N1 influenza virus clinical isolates. Sci Rep. (2018) 8:13066. doi: 10.1038/s41598-018-31397-3

53. Venkatesh D, Bianco C, Nunez A, Collins R, Thorpe D, Reid SM, et al. Detection of $\mathrm{H} 3 \mathrm{~N} 8$ influenza A virus with multiple mammalian-adaptive mutations in a rescued Grey seal (Halichoerus grypus) pup. Virus Evol. (2020) 6:veaa016. doi: 10.1093/ve/veaa016

54. Daniloski Z, Jordan TX, Ilmain JK, Guo X, Bhabha G, tenOever BR, et al. The Spike D614G mutation increases SARS-CoV-2 infection of multiple human cell types. bioRxiv. (2020). doi: 10.1101/2020.06.14.151357

55. Eaaswarkhanth M, Al Madhoun A, Al-Mulla F. Could the D614G substitution in the SARS-CoV-2 spike (S) protein be associated with higher COVID-19 mortality? Int J Infect Dis. (2020) 96:459-60. doi: 10.1016/j.ijid.2020.05.071

56. Hu J, He CL, Gao QZ, Zhang J, Cao XX, Long QX, et al. The D614G mutation of SARS-CoV-2 spike protein enhances viral infectivity and decreases neutralization sensitivity to individual convalescent sera. bioRxiv. (2020). doi: 10.1101/2020.06.20.161323

57. Zhang L, Jackson CB, Mou H, Ojha A, Rangarajan ES, Izard T, et al. The D614G mutation in the SARS-CoV-2 spike protein reduces S1 shedding and increases infectivity. bioRxiv. (2020). doi: 10.1101/2020.06.12. 148726

58. Emma CT, Laura ER, James GS, Roberto S, Ana SF, Jason AW, et al. Circulating SARS-CoV-2 spike N439K variants maintain fitness while evading antibody-mediated immunity. Cell. (2021). doi: 10.1016/j.cell.2021. 01.037

Conflict of Interest: The authors declare that the research was conducted in the absence of any commercial or financial relationships that could be construed as a potential conflict of interest.

Copyright (c) $2021 \mathrm{Liu}$, Zeng, Rao, Yuan, Ji, Lei, Xiao, Li, Li, Du, Liu, Tan, Li, Zhu, Yang and Liu. This is an open-access article distributed under the terms of the Creative Commons Attribution License (CC BY). The use, distribution or reproduction in other forums is permitted, provided the original author(s) and the copyright owner(s) are credited and that the original publication in this journal is cited, in accordance with accepted academic practice. No use, distribution or reproduction is permitted which does not comply with these terms. 